\title{
Clinical characteristics of breast abscesses induced by methicillin-resistant Staphylococcus aureus during lactation
}

Yan Li ( $\square$ liyan1984311@126.com )

Maternal and Child Health Hospital of Haidian District https://orcid.org/0000-0002-4901-2817

\section{Xiangjun Ma}

Maternal and Child Health Hospital of Haidian District

\section{Xingping He}

Maternal and Child Health hospital of Haidian District

\section{Research}

Keywords: MRSA, Staphylococcus aureu, Breast abscess, Lactation, Puncture for abscess

Posted Date: October 26th, 2020

DOI: https://doi.org/10.21203/rs.3.rs-80947/v1

License: (c) (i) This work is licensed under a Creative Commons Attribution 4.0 International License.

Read Full License 


\section{Abstract \\ Background}

This study aims to identify the differences in patients with breast abscesses in combination with the infection of methicillin-resistant Staphylococcus aureus (MRSA) or with methicillin-susceptible Staphylococcus aureus (MSSA) during lactation in the clinical characteristics, efficacy of puncture, antibiotic use, treatment duration, breastfeeding after illness and recurrence.

\section{Methods}

Clinical data of patients with breast abscesses during lactation who were treated in our hospital from January 2014 to February 2017 were reviewed. According to bacterial culture results, they were divided into MRSA $(n=260)$ and MSSA $(n=962)$ groups. The hospitalisation, postpartum time, age, location of abscess cavities, number of abscess cavities, maximum amount of punctured pus, frequency of punctures, failure of puncture, antibiotic use, treatment duration, delactation and recurrence were compared between groups using a t-test and a chi-squared test.

\section{Results}

The statistical analysis showed that only delactation was statistically significantly different between the groups $(P=0.018)$. Hospitalisation, postpartum time, age, location of abscess cavities, number of abscess cavities, maximum amount of punctured pus, frequency of punctures, failure of puncture, antibiotic use, treatment duration and recurrence showed no statistically significant differences $(P=$ $0.488, P=0.328, P=0.494, P=0.218, P=0.088, P=0.102, P=0.712, P=0.336, P=0.512, P=0.386$ and $P$ $=0.359$, respectively).

\section{Conclusion}

Patients with breast abscesses combined with MRSA infection during lactation presented no significant differences in the clinical manifestations, efficacy of puncture, antibiotic use or treatment duration from those with MSSA infection. However, the patients infected with MRSA were more susceptible to delactation after illness.

\section{Introduction}

Breast abscesses are common in lactating women, with an incidence of $0.4-11 \%$ during lactation [1]. Staphylococcus aureus is the most common pathogenic bacteria among breast abscesses during lactation. As a Gram-positive bacteria, it has strong pathogenicity and can cause skin and soft-tissue, bone and joint, and systemic organ infections [2-5]. In 1961, the world's first case of methicillin-resistant 
Staphylococcus aureus (MRSA) was isolated [6]. MRSA is characterised by high-level drug resistance and a complex drug resistance mechanism, which can increase infection-caused mortality, prolong the length of the hospital stay and increase medical expenses [7]. In recent years, the detection rate of MRSA in the pus of breast abscesses has gradually increased. Whether the condition of patients with breast abscesses combined with MRSA infection is more serious than those infected with methicillin-susceptible Staphylococcus aureus (MSSA) or whether MRSA increases the difficulty of breast abscess treatment remains unclear. Presently, there are few studies on these aspects, and the sample sizes were small. Therefore, we intended to determine the clinical manifestations, efficacy of puncture, antibiotic use, treatment duration, breastfeeding after illness and recurrence of patients with breast abscesses combined with MRSA or MSSA infection during lactation, using a large sample study, to guide the clinical diagnosis and treatment.

\section{Materials And Methods}

\section{Patient data and criteria}

This was a retrospective study. We collected the clinical data on patients with breast abscesses during lactation treated in the Breast Disease Prevention and Treatment Centre of Haidian Maternal and Child Health Hospital, Beijing, from January 2014 to February 2017, using the electronic medical record system and reviewing medical records in the medical record room. According to their bacterial culture results, all the patients were assigned to either the MRSA or MSSA group. Hospitalisation, postpartum time, age, location of abscess cavities, number of abscess cavities, maximum amount of punctured pus, frequency of punctures, treatment method (failure of puncture), antibiotic use, treatment duration, delactation and recurrence were compared between the two groups. We aimed to determine whether the condition of patients with breast abscesses induced by MRSA infection was more serious than of those with MSSA infection and whether MRSA infection would prolong treatment duration or increase the recurrence rate.

Inclusion criteria: (1) Patients diagnosed as having breast abscesses during lactation who met all of the following criteria: lactating woman; breast lesions with inflammatory manifestations such as redness, swelling, heat and pain or accompanied by fever; physical examination showing touched fluctuation; breast ultrasound suggesting mixed echo or anechoic areas in the breast and liquid flow observed after probe pressurisation and pus aspirated by puncture. (2) The patients' pus was examined via bacterial culture. (3) The results of the bacterial culture showed MRSA or MSSA.

Exclusion criteria: (1) The patients had no MRSA or MSSA, or there was no bacterial growth in the bacterial culture $(n=259)$; $(2)$ the patients were lost to follow-up $(n=24)$.

Cure criteria of puncture: The patients' clinical symptoms disappeared, and the fluid aspirated by the last puncture was non-purulent.

Failure criteria of puncture: Skin ulceration occurred after puncture, or the clinical symptoms were not relieved after puncture, and the patients were transferred to surgery. 
Recurrence criteria: All the patients followed up for 1 month without the recurrence of clinical symptoms or another puncture were considered clinically cured. Otherwise, the case was regarded as a recurrence.

A total of 1525 patients with breast abscesses during lactation were treated in the Breast Disease Prevention and Treatment Centre of Haidian Maternal and Child Health Hospital, Beijing, from January 2014 to February 2017, among which 20 patients received no bacterial culture, 24 were lost to follow-up and 259 showed non-MRSA or non-MSSA in the bacterial culture. Thus, a total of 1222 patients met the inclusion criteria.

This study was approved by the ethics committee of Haidian Maternal and Child Health Hospital, Beijing.

\section{Treatment methods}

All the patients were treated with ultrasound-guided fine-needle puncture and irrigation for abscesses. If the clinical symptoms were not relieved after the puncture, sensitive antibiotic adjuvant therapy was selected according to the drug sensitivity results of the bacterial culture. If the clinical symptoms were still not relieved, surgical treatment was considered. Surgical methods included Mammotome minimally invasive vacuum-assisted biopsy (VAB) of the abscess, abscess catheter irrigation and drainage and abscess incision and drainage(Table 1).

Ultrasound-guided fine-needle puncture and irrigation: after local anaesthesia, puncture was conducted with a $20 \mathrm{ml}$ syringe through the located puncture point under ultrasound guidance. After aspirating pus, the abscess cavity was repeatedly washed with normal saline until the flushing fluid became clear. A bacterial culture and drug sensitivity test were conducted for the aspirated pus. Repeated puncture was determined according to the patients' condition and ultrasonic re-examination 1-2 days after the puncture.

Mammotome minimally invasive vacuum-assisted biopsy of the abscess: after local anaesthesia, the skin was cut open approximately $3-5 \mathrm{~mm}$ in length at the predetermined puncture point, and the rotary cutter was inserted into the abscess cavity to aspirate the pus, destroy the septum of the abscess cavity and remove the necrotic tissue from the cavity. Next, the abscess cavity was repeatedly washed with normal saline until the flushing fluid became clear. A silica gel drainage tube with a $5 \mathrm{~mm}$ diameter was placed in the abscess cavity along the incision, and a $20 \mathrm{ml}$ negative pressure syringe was fixed and connected. The wound dressing was changed every other day, and the drainage tube was removed approximately 5-7 days later. (The specific time was determined according to the patient's drainage volume. Generally, the drainage tube was removed when drainage volume was $<5 \mathrm{ml}$ ).

Abscess catheter irrigation and drainage: after intravenous general anaesthesia, a skin incision of 1.5$2.0 \mathrm{~cm}$ in length was cut from the lowest point of the abscess cavity. The subcutaneous tissue was bluntly separated with curved forceps till the abscess cavity to drain the pus, separate the septum in the cavity and remove the necrotic tissue. An infusion tube was inserted into the top of the abscess cavity, connected with $500 \mathrm{ml}$ normal saline, to repeatedly wash the cavity. Another silica gel drainage tube with a side hole was inserted into the abscess cavity 2.0-3.0 away from the incision, connected with a sterile 
drainage bag. The irrigation tube and drainage tube were sutured and fixed on the skin incision with silk thread. On the first day after surgery, the irrigation tube was opened every $6 \mathrm{~h}$ for flushing. Afterwards, the flushing interval was gradually prolonged. When no obvious necrotic tissue was discharged with flushing fluid, the irrigation tube and drainage tube could be withdrawn, and the drainage strip was placed in the residual cavity until the abscess cavity and incision were completely healed.

Abscess incision and drainage: after successful local anaesthesia, an incision was made at the abscess, and the pus and necrotic tissue were removed after reaching the abscess cavity. The abscess cavity was then repeatedly washed with normal saline until no obvious pus or necrotic tissue remained. The drainage strip was placed into the incision, and the dressing and drainage strip were changed every day until no pus was discharged. Afterwards, the dressing was changed intermittently (every 2-3 d) until the incision and abscess cavity were healed.

\section{Follow-up}

All the patients were followed up for 1 month by regular outpatient treatment and telephone follow-up.

\section{Statistical analysis}

The data were statistically processed using SPSS 21.0. Hospitalisation, postpartum time, location of abscess cavities, number of abscess cavities, failure of puncture, antibiotic use, delactation and recurrence were analysed using the chi-squared test. Age, maximum amount of punctured pus, frequency of punctures and treatment duration were analysed using a t-test.

\section{Results}

Of the 1525 patients with breast abscesses during lactation we reviewed, 1481 received a bacterial culture of pus (20 of 1525 patients did not receive a bacterial culture and 24 were lost to follow-up and were excluded). The results showed 260 cases of MRSA, 962 cases of MSSA, seven cases of methicillinresistant Staphylococcus epidermidis, 13 cases of Staphylococcus epidermidis, and 42 cases of other bacteria, including Streptococcus tarsus, Streptococcus sanguinis and Staphylococcus intermedia. No growth of pathogenic bacteria was detected in the bacterial cultures of the other 197 patients. A total of 1222 patients met the inclusion criteria. According to their bacterial culture results, they were assigned to the MSSA or MRSA group and aged 21-44 years (mean age, 30.57 years).

All 1222 patients with breast abscesses during lactation were analysed using the chi-squared test, revealing a statistically significant difference in delactation between the groups $\left(\chi^{2}=5.290, P=0.018\right)$. No statistically significant differences were found in hospitalisation $\left(\chi^{2}=0.012, P=0.488\right)$, postpartum time $\left(\chi^{2}=0.269, P=0.328\right)$, location of abscess cavities $\left(\chi^{2}=0.738, P=0.218\right)$, number of abscess cavities $\left(\chi^{2}\right.$ $=2.063, P=0.088)$, failure of puncture $\left(\chi^{2}=0.331, P=0.336\right)$, antibiotic use $\left(\chi^{2}=0.003, P=0.512\right)$ or recurrence $\left(\chi^{2}=0.390, P=0.359\right)$ between the groups (Table 2$)$. The t-test demonstrated that the differences in age $(P=0.494)$, maximum amount of punctured pus $(P=0.102)$, frequency of punctures $(P$ 
$=0.712)$ and treatment duration $(P=0.386)$ were not statistically significant between the groups (Table 2 , Table 3).

\section{Discussion}

Since the first isolation of MRSA by Jevons in 1961 [6], the infection and isolation rates of MRSA have been increasing worldwide, and it has become one of the primary pathogens of nosocomial and community-acquired infections $[8,9]$. Annually, the number of deaths caused by MRSA infection in the United States is equivalent to the deaths caused by AIDS, tuberculosis and viral hepatitis combined [10]. The prevalence of MRSA infection has become a serious clinical and public health problem.

In recent years, the detection rate of MRSA in the breast milk and pus of lactating patients has gradually increased[2, 3]. Stafford I and Moazzez A et al. [2, 3] found that the detection rate of MRSA in patients with breast abscesses and mastitis was $67 \%$ and $58 \%$, respectively. In our study, of 1481 patients receiving a bacterial culture of pus, 260 cases of MRSA were detected, with a detection rate of $17.56 \%$, making our detection rate lower than that of Stafford I and Moazzez A et al. The sample sizes in the studies of Stafford I and Moazzez A et al. were small, at 35 and 44, respectively, which are far smaller than our sample size. This difference could explain our lower detection rate than that of these two studies. Although the infection rate of MRSA in lactating patients is increasing, the cause of the infection remains unclear. Some scholars speculated that patients need hospitalisation before and after delivery, which could increase the probability of MRSA infection, but further research is required to confirm this [11-14].

In our study, whether the patients were hospitalised was used as an indicator to evaluate the patients' condition, to compare the severity in the two groups. Evaluating the postpartum time (puerperium), patients' age, location of abscess cavities, number of abscess cavities and maximum amount of punctured pus, the clinical manifestations of the patients infected with the two bacteria were compared. Furthermore, the therapeutic effect of the frequency of punctures, efficacy of puncture, treatment duration and antibiotic use was evaluated. Additionally, the prognosis of the patients was evaluated according to whether the patients had delactation and recurrence. Therefore, we divided the above factors into three aspects for discussion: clinical manifestations, therapeutic effect and prognosis.

\section{Clinical manifestations}

Reddy et al. [15] compared MRSA- and MSSA-infected patients with postpartum mastitis, showing no significant differences in age, pregnancy history, initial symptoms or mode of delivery between the patients infected with the two bacteria. Chen $\mathrm{CY}$ et al. [16] studied patients with breast abscesses combined with MRSA or MSSA infection, which suggested that MRSA infection did not increase the hospitalisation rate of patients with breast abscesses. The results of our study showed no significant differences in hospitalisation, postpartum time or age between the MRSA and the MSSA groups, which is consistent with the above results. 
Studies have shown that central breast abscesses, multi-cavity abscesses and abscesses $>5 \mathrm{~cm}$ are complex and refractory abscesses[17-19], which increase the difficulty of treatment. In our study, no significant differences were found in the location of the abscess, the number of abscess cavities or the amount of punctured pus between patients with breast abscesses combined with MRSA infection or MSSA infection during lactation, which did not increase the difficulty of treatment. Although the results of our study were inconsistent with the above results, the sample size of the above studies was smaller than that of our samples and included some nonlactating breast abscess cases[19], which could have led to inconsistent results.

Therefore, based on our study, the clinical manifestations of patients with breast abscesses combined with MRSA infection during lactation were not different from those with MSSA infection, and MRSA infection did not increase the hospitalisation rate, indicating that MRSA infection did not increase the severity of the breast abscess or the difficulty of treatment.

\section{Therapeutic effect}

With the development of minimally invasive treatment technology, ultrasound-guided fine-needle puncture and irrigation has become the preferred treatment method for breast abscesses and is widely used in clinical practice ${ }^{[20,21]}$. Chen $\mathrm{CY}$ et al.[16] showed that MRSA infection did not reduce the therapeutic effect of ultrasound-guided percutaneous drainage or prolong the duration of treatment and antibiotic use compared with MSSA infection in postpartum breast abscess. In our study, 1222 patients were treated with ultrasound-guided fine-needle puncture and irrigation. Of these, 90 failed in the puncture, including abscess ulceration after puncture or ineffective puncture, and then, they underwent Mammotome minimally invasive vacuum-assisted biopsy, abscess catheter irrigation and drainage or abscess incision and drainage. However, the statistical analysis showed no difference in the therapeutic effect of fine-needle puncture between the MRSA and MSSA groups, and MRSA infection did not increase the failure rate of fine-needle puncture. The mean frequency of puncture in the MRSA and MSSA groups was 3.0 and 2.9, respectively, without statistical significance, which also indicated that MRSA did not increase the frequency of abscess puncture. Among the 1222 patients, the treatment duration was 2-82 days, with a mean of 8 days. The treatment duration was 7.9 days in the MRSA group and 8 days in the MSSA group, without statistically significant differences, indicating that MRSA infection did not prolong the treatment duration of patients with breast abscesses during lactation. The above results are consistent with those of Chen $\mathrm{CY}$ et al[6]. Therefore, when clinicians treat MRSA-infected patients with breast abscesses during lactation, abscess puncture remains the first choice. Additionally, compared with MSSA infection, MRSA infection did not increase the frequency of puncture or treatment duration of these patients.

According to Jiayue Luo et al. [22], antibiotics were not routinely used for the treatment of breast abscesses during lactation, but the success rate was similar to that of routine antibiotic use. Thus, they are conducting a prospective randomised controlled study on whether breast abscesses during lactation should be treated with antibiotics during drainage. The participants were randomly divided into two 
groups: an antibiotic group (antibiotic use $5 \mathrm{~d}$ after surgery) and a nonantibiotic group (no antibiotic use after surgery). The authors will observe the treatment duration and recurrence rate of breast abscess. Their expected conclusion is that for the treatment of breast abscesses, the effect of drainage without antibiotics is not poorer than that of drainage combined with antibiotics. However, the study has not yet been completed, and the expected conclusion has not yet been confirmed. In our study, symptoms were significantly relieved after abscess puncture or drainage, without antibiotic use. However, for the patients with severe systemic symptoms or poor effects of the puncture, antibiotics were administered. Among the 260 patients with MRSA infection, 82 were treated with antibiotics and the remaining 178 were not. Among the 82 patients receiving antibiotics, two were treated with vancomycin and the rest with levofloxacin. Of the 962 patients with MSSA infection, 305 were treated with antibiotics and 657 were not. The statistical analysis demonstrated no difference between the two groups, suggesting that MRSA infection in breast abscesses during lactation did not increase the use of antibiotics. All the patients without antibiotic treatment were cured. Therefore, for the treatment of breast abscesses during lactation, effective drainage of pus might be necessary. Without drainage of infected fluid, the use of antibiotics is ineffective [23].

\section{Prognosis}

The study by Reddy et al. [15] showed that the delactation rate after mastitis in the MRSA group was $16 \%$, and the MSSA group 22\%, without statistical significance. However, our results showed that only delactation was significantly different between patients with breast abscesses during lactation in the MRSA group and the MSSA group. Among the 1222 patients, 28 in the MRSA group presented delactation, with a rate of $10.7 \%(28 / 260)$, whereas $63(6.5 \%, 63 / 962)$ patients in the MSSA group showed delactation. The rate of delactation in the MRSA group was higher than that in the MSSA group, which indicated that patients with breast abscesses infected with MRSA during lactation were more susceptible to delactation after illness than those infected with MSSA and could not continue to breastfeed. The authors believe that this is related to the patients' concern that MRSA infection would prolong the recovery time and their desire to prevent the recurrence of the abscess by delactation. Furthermore, delactation is also related to the mothers' concern that MRSA could be transmitted to the child through her milk, leading to adverse effects for the child. These concerns increase the delactation rate of patients with breast abscesses infected with MRSA during lactation. A study in Taiwan [24] reported that the colonisation rate of MRSA in the nasal cavity of healthy children was $8.1 \%$, and breastfeeding could prevent the colonisation of MRSA and MSSA in healthy children, which might be related to improvements in children's immunity by breastfeeding. Therefore, breastfeeding can still be continued in patients with breast abscesses infected with MRSA during lactation, and delactation is related to the subjective concerns of the patients. Acute mastitis and breast abscesses during lactation do not affect breastfeeding. The Academy of Breastfeeding Medicine [25] suggests that the first step in the treatment of mastitis during lactation is to effectively remove the milk; that is, encourage the mothers to breastfeed frequently and start from the affected side. If breastfeeding by the patients with breast abscesses during lactation is affected before treatment, it should be recovered as soon as possible after drainage to prevent galactostasis and infection recurrence. Additionally, Irusen $\mathrm{H}$ et al. recommended that 
mothers with breast abscesses during lactation should feed with the infected breast but that the baby's mouth should not be exposed to the infected liquid or breast tissue [23]. Our results showed that patients in the MRSA group were more susceptible to delactation after illness than those in the MSSA group, which differs from the findings of Reddy et al. [15]. This result might indicate the defect of doctors' work in our department; that is, the proper education of patients about the condition is insufficient. Patients had a poor understanding of the disease and MRSA and had a fear of MRSA, thus leading to an increased delactation rate in the MRSA group. Therefore, we should also consider the psychological burden on the patients while undergoing treatment, so that the patients can correctly understand the disease and MRSA and eliminate their concerns, which might change the outcome.

In our study, a total of 30 patients had abscess recurrence, including five in the MRSA group and 25 in the MSSA group, without a significant difference, which is consistent with the results of Chen CY et al. [16]. Their study showed that MRSA infection did not increase the hospitalisation rate, the frequency of outpatient follow-up or the recurrence rate compared with MSSA infection in postpartum breast abscess.

In conclusion, the infection and isolation rates of MRSA are increasing globally. Given the multi-drug resistance mechanism of MRSA, clinicians are more alert to these bacteria. Therefore, many doctors might believe that patients with breast abscesses infected by MRSA during lactation have a more serious condition and are difficult to treat. In terms of treatment options, they will intervene more actively for MRSA than for an MSSA infection. However, our study showed that the age, postpartum time, location of abscess cavities, number of abscess cavities, frequency of punctures, effect of puncture, treatment duration and recurrence were not significantly different between patients with breast abscesses that were infected by MRSA during lactation and those infected by MSSA, suggesting that MRSA infection does not increase the severity of breast abscess or the difficulty of treatment. For breastfeeding patients with breast abscesses, whether infected with MRSA or not, ultrasound-guided abscess puncture and irrigation should be the first-choice treatment[16]. For antibiotic use, if ultrasound-guided fine-needle puncture has a good effect in the patients with breast abscesses infected by MRSA during lactation, antibiotics are not necessary. If the treatment effect is not good, it can be combined with antibiotic use. According to the results of the bacterial culture, drugs sensitive to MRSA can be used without overtreatment.

\section{Conclusion}

Our study has shown that the condition and prognosis of patients with breast abscesses combined with MRSA infection during lactation were not poorer than those with MSSA infection, and no special treatment was required. However, to avoid the spread of MRSA, we suggest that the inpatients with MRSA infection should be placed in isolation wards, outpatient clinics should be disinfected routinely and doctors should wash and disinfect hands strictly according to the principle of nosocomial infection prevention and control. Furthermore, although the sample size in this study was large, it was a retrospective study and has some limitations. Therefore, the results should be further confirmed by large sample prospective studies. 


\section{Abbreviations}

MRSA: methicillin-resistant Staphylococcus aureus; MSSA: methicillin-susceptible Staphylococcus aureus.

\section{Declarations}

\section{Acknowledgments}

Not applicable.

\section{Authors' contributions}

YL drafted the manuscript with the help of XJM and XPH. XJM critically revised and supervised the study. All authors read and approved the final manuscript.

\section{Funding}

None.

\section{Availability of data and materials}

The datasets used and/or analyzed during the current study are available

from the corresponding author on reasonable request.

\section{Ethics approval and consent to participate}

Neither medical nor ethical approval was required. Our study was a retrospective study. All data are derived from the medical records of patients who have been treated. And all data were processed anonymously.

\section{Consent for publication}

Not applicable.

\section{Competing interests}

The authors declare that they have no competing interests.

\section{References}

1. Kataria K, Srivastava A, Dhar A. Management of lactational mastitis and breast abscesses: review of current knowledge and practice. Indian J Surg. 2013;75:430-5. 
2. Stafford I, Hernandez J, Laibl V, Sheffield J, Roberts S, Wendel G Jr. Community-acquired methicillinresistant Staphylococcus aureus among patients with puerperal mastitis requiring hospitalization. Obstet Gynecol. 2008;112:533-7.

3. Moazzez A, Kelso RL, Towfigh S, Sohn H, Berne TV, Mason RJ. Breast abscess bacteriologic features in the era of community-acquired methicillin-resistant Staphylococcus aureus epidemics. Arch Surg. 2007;142:881-4.

4. Soltau $\mathrm{DH}$, Hatcher GW. Some observations on the aetiology of breast abscess in the puerperium. $\mathrm{Br}$ Med J. 1960;1:1603-7.

5. Dener C, Inan A. Breast abscesses in lactating women. World J Surg. 2003;27:130-3.

6. Barber M. Methicillin-resistant staphylococci. J Clin Pathol. 1961;14:385-93.

7. Miura Y, Yamaguchi T, Nakamura I, et al. Epidemiological trends observed from molecular characterization of methicillin-resistant Staphylococcus aureus isolates from blood cultures at a Japanese University Hospital, 2012-2015[J]. Microb Drug Resist. 2018;24(1):70-5.

8. Matouskova I, Janout V. Current knowledge of methicillin-resistant Staphylococcus aureus and community-associated methicillinresistant Staphylococcus aureus. Biomed Pap Med Fac Univ Palacky Olomouc Czech Repub. 2008;152(2):191-202.

9. Diekema DJ, Pfaller MA, Schmitz FJ,et al. Survey of infections due to Staphylococcus species: frequency of occurrence and antimicrobial susceptibility of isolates collected in the United States, Canada, Latin America, Europe, and the Western Pacific region for the Sentry Antimicrobial Surveillance Program, 1997-1999. Clin Infect Dis. 2001;32(Suppl2):114-32.

10. Boucher HW, Corey GR. Epidemiology of methicillin-resistant Staphylococcus aureus. Clin Infect Dis. 2008;46(Supp15):344-9.

11. Boccaccio C, Verdaguer Babic V, Botto L, et al. Methicillin-resistant Staphylococcus aureus (MRSA) isolation in breast abscesses in a Public Maternity. Medicina. 2014;74:210-5.

12. Dabbas N, Chand M, Pallett A, Royle GT, Sainsbury R. Have the organisms that cause breast abscess changed with time?- Implications for appropriate antibiotic usage in primary and secondary care. Breast J. 2010;16:412-5.

13. Chuwa EW, Wong CM, Tan YY, Hong GS. MRSA breast abscesses in postpartum women. Asian J Surg. 2009;32:55-8.

14. Berens P, Swaim L, Peterson B. Incidence of methi- cillin-resistant Staphylococcus aureus in postpartum breast abscesses. Breastfeed Med. 2010;5:113-5.

15. Reddy P, Qi C, Zembower T, Noskin GA, Bolon M. Postpartum mastitis and community-acquired methicillin-resistant Staphylococcus aureus. Emerg Infect Dis. 2007;13:298-301.

16. Chen CY, Anderson BO, Lo SS, et al. Methicillin-resistant Staphylococcus aureus infections may not impede the success of ultrasound-guided drainage of puerperal breast abscesses. J Am Coll Surg. 2010;210:148-54. 
17. Gao HF, Ma XJ, Wang J, et al. The initial study of the effect of the treatment with dexamethasone of mastitis in the central area in breast-feeding. China J Mod Med. 2011;21(34):4337-40.

18. Gao YJ, Ma XJ, He XP, et al. Analysis of the clinical features and treatment of the central area of the lactation mastitis. Chin J Gen Pract. 2011;8:591-2.

19. Lam E, Chan T, Wiseman SM. Breast abscess: evidence based management recommendations. Expert Rev Anti Infect Ther,2014,12(7):753-762.

20. Elagili $F$, Abdullah $N$, Fong $L$, et al. Aspiration of breast abscess under ultrasound guidance: outcome obtained and factors affecting success. Asian J Surg. 2007;30(1):40-4.

21. Elder EE, Brennan M. Nonsurgical management should be first line therapy for breast abscess. World J Surg,2010,34(9):2257-2258.

22. Luo J, Long T, Cai Y, et al. Abscess Drainage with or Without Antibiotics in Lactational Breast Abscess: Study Protocol for a Randomized Controlled Trial. Infect Drug Resist. 2020;13:183-90.

23. Irusen H, Rohwer AC, Steyn DW, Young T. Treatments for breast abscesses in breastfeeding women. Cochrane Database Syst Rev. 2015;8:Cd010490.

24. Chen $\mathrm{CH}$, Kuo KC, Hwang KP, Lin TY, Huang YC. Risk factors for and molecular characteristics of methicillin-resistant Staphylococcus aureus nasal colonization among healthy children in southern Taiwan, 2005-2010. J Microbiol Immunol Infect. 2019Dec;52(6):929-36.

25. Amir LH, The Academy of Breastfeeding Medicine Protocol Committee. ABM Clinical Protocol \#4: Mastitis, Revised. March 2014. Breastfeeding Medicine. 2014;9(5):239-43.

\section{Tables}


Table 1. Flow chart of treatment of breast abscess during lactation

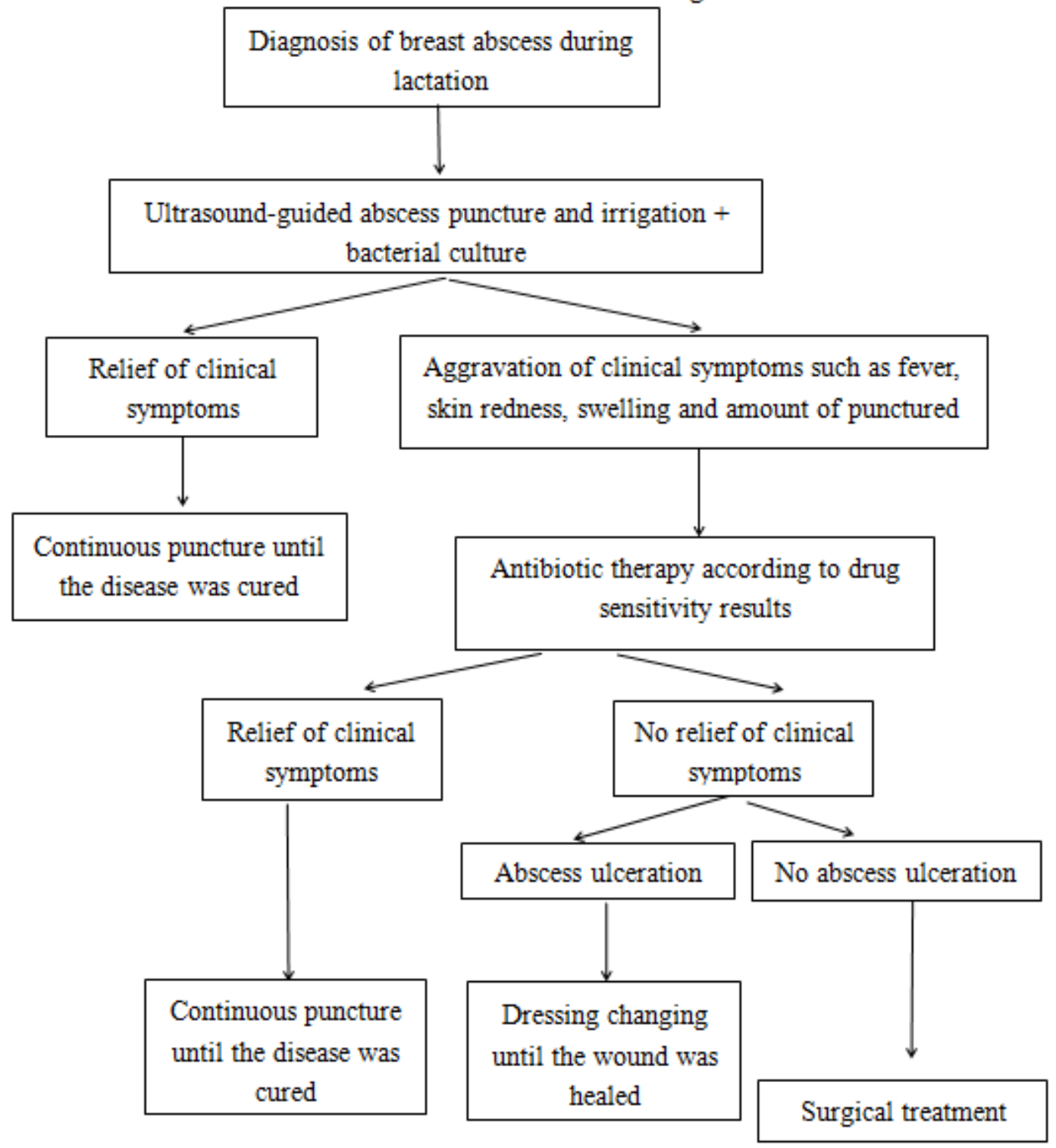

Table 2. Factor analysis results by chi-squared test 


\begin{tabular}{|c|c|c|c|c|}
\hline Factors & $\begin{array}{l}\text { MRSA group } \\
(n=260)\end{array}$ & $\begin{array}{l}\text { MSSA group } \\
(\mathrm{n}=962)\end{array}$ & $x^{2}$ & $P$ value \\
\hline Hospitalisation & & & 0.012 & 0.488 \\
\hline Residential treatment & 44 & 160 & & \\
\hline Outpatient treatment & 216 & 802 & & \\
\hline Postpartum time & & & 0.269 & 0.328 \\
\hline Puerperium & 149 & 534 & & \\
\hline Non-puerperium & 111 & 428 & & \\
\hline Location of abscess cavities & & & 0.738 & 0.218 \\
\hline Central area & 66 & 270 & & \\
\hline Non-central area & 194 & 692 & & \\
\hline Number of abscess cavities & & & 2.063 & 0.088 \\
\hline Single abscess cavity & 180 & 709 & & \\
\hline Multiple abscess cavities & 80 & 253 & & \\
\hline Failure of puncture & & & 0.331 & 0.336 \\
\hline Failed & 17 & 73 & & \\
\hline Non-failed & 243 & 889 & & \\
\hline Antibiotic use & & & 0.003 & 0.512 \\
\hline Yes & 82 & 305 & & \\
\hline No & 178 & 657 & & \\
\hline Delactation & & & 5.290 & 0.018 \\
\hline Delactation & 28 & 63 & & \\
\hline Non-delactation & 232 & 899 & & \\
\hline Recurrence & & & 0.390 & 0.359 \\
\hline Recurrent & 5 & 25 & & \\
\hline Non-recurrent & 255 & 937 & & \\
\hline
\end{tabular}

This is the most parts of data of the patients in the study and the factor analysis results by chi-squared test.

Table 3. Factor analysis results by t-test 


\begin{tabular}{|llll|}
\hline Factor & $\begin{array}{l}\text { MRSA group } \\
(\mathrm{n}=260)\end{array}$ & $\begin{array}{l}\text { MSSA group } \\
(\mathrm{n}=962)\end{array}$ & P value \\
\hline Mean age (years) & $30.6(21-44)$ & $30.6 \otimes 20-48)$ & 0.494 \\
Mean amount of punctured pus $(\mathrm{ml})$ & $16.5(0-143)$ & $17.9(0-420)$ & 0.102 \\
Mean frequency of punctures (times) & $3.0(0-12)$ & $2.9(0-16)$ & 0.712 \\
Mean treatment duration $\llbracket \mathrm{d} \rrbracket$ & $7.9(1-90)$ & $7.8(1-85)$ & 0.386 \\
\hline
\end{tabular}

This is a apart of data of the patients in the study and the factor analysis results by t-test 